\title{
A Two-grid Method for Coupled Free Flow with Porous Media Flow
}

\author{
Prince Chidyagwai $^{\mathrm{a}}$ and Béatrice Rivière ${ }^{\mathrm{a}, 1}$ \\ ${ }^{a}$ Department of Computational and Applied Mathematics, Rice University, 6100 \\ Main Street, Houston, TX 7r005, U.S.A.
}

\begin{abstract}
This paper presents a two-grid method for solving systems of partial differential equations modelling free flow coupled with porous media flow. This work considers both the coupled Stokes and Darcy as well as the coupled Navier-Stokes and Darcy problems. The numerical schemes proposed are based on combinations of the continuous finite element method and the discontinuous Galerkin method. Numerical errors and convergence rates for solutions obtained from the two-grid method are presented. CPU times for the two-grid algorithm are shown to be significantly less than those obtained by solving the fully coupled problem.
\end{abstract}

Key words: Navier-Stokes equations, Darcy's law, discontinuous Galerkin, finite element method, Beavers-Joseph-Saffman, decoupled and linearized problems, efficiency.

\section{Introduction}

The coupling of free flow with porous media flow has been studied extensively in the literature. Interest in this coupling is mostly due to the wide range of applications for example in biosciences, geosciences and industrial applications. The Navier-Stokes and Darcy coupling has been analyzed for example in $[1,2,3,4]$. The Stokes and Darcy model has been studied for example in

$\overline{1}$ The second author acknowledges the support of NSF through the grant DMS 0810422 . 
$[5,6,7,8,9,10]$. The coupled Navier-Stokes and Darcy schemes studied in $[1,4]$ result in large non-linear coupled systems which may be time consuming to solve. In this paper, we apply the two-grid technique for both the coupled Stokes/Darcy and the Navier-Stokes/Darcy problems. We show that the twogrid method is attractive for these systems because it decouples the coupled model into two relatively smaller problems. The first is an Oseen problem (or Stokes problem) to solve for the fluid velocity and pressure in the free flow domain. The second is a modified Darcy problem to solve for the Darcy pressure in the porous medium. The two grid technique has several advantages. First it allows for easy parallelization. Second, legacy codes exist that have been optimized to solve the Stokes, Navier-Stokes or Darcy problems separately. These codes can be used independently with the two-grid approach. In this work, we use a multi-numerics scheme that combines the continuous finite element method with the discontinuous Galerkin method. The use of the latter in the subsurface offers many advantages: local mass conservation, local and high order accuracy, robustness of the method with respect to the heterogeneities of the medium. Recently, the two-grid method applied to a first order continuous finite element method is analyzed for solving the Stokes/Darcy [11] and Navier-Stokes/Darcy problems [12].

An outline of the paper is as follows. The mathematical model and its fully coupled numerical discretization are described in Section 2 and Section 3 respectively. The two-grid method is introduced in Section 4. It yields decoupled, linearized and smaller problems to solve. Numerical examples are shown in Section 5. Conclusions follow.

\section{Fluid Flow Models}

Let $\Omega \subset \mathbb{R}^{d}$ be a bounded polygonal domain subdivided into a finite number of non-overlapping subdomains $\Omega_{1}^{i}$ and $\Omega_{2}^{i}$. The free flow domain is $\Omega_{1}=\cup_{i} \Omega_{1}^{i}$ in which the flow is modelled by the Stokes or Navier-Stokes equations. The domain $\Omega_{2}=\cup_{i} \Omega_{2}^{i}$ is the porous medium region where the flow is described by Darcy's Law. The free flow and porous media domains are separated by a polygonal interface $\Gamma_{12}$. First we state the equations that govern the flow in each domain. The flow in $\Omega_{1}$ can be described by either the Stokes equations:

$$
\begin{aligned}
& -\nabla \cdot\left(2 \nu \boldsymbol{D}\left(\boldsymbol{u}_{1}\right)\right)+\nabla p_{1}=\boldsymbol{f}_{1}, \quad \text { in } \Omega_{1}, \\
& \nabla \cdot \boldsymbol{u}_{1}=0, \quad \text { in } \Omega_{1} \text {, } \\
& \boldsymbol{u}_{1}=0, \quad \text { on } \Gamma_{1}=\partial \Omega \cap \partial \Omega_{1} \text {, }
\end{aligned}
$$

or the Navier-Stokes equations: 


$$
\begin{array}{rc}
-\nabla \cdot\left(2 \nu \boldsymbol{D}\left(\boldsymbol{u}_{1}\right)\right)+\nabla p_{1}+\boldsymbol{u}_{1} \cdot \nabla \boldsymbol{u}_{1}=\boldsymbol{f}_{1}, & \text { in } \Omega_{1}, \\
\nabla \cdot \boldsymbol{u}_{1}=0, & \text { in } \Omega_{1}, \\
\boldsymbol{u}_{1}=0, & \text { on } \Gamma_{1} .
\end{array}
$$

The fluid velocity and pressure in $\Omega_{1}$ are denoted by $\boldsymbol{u}_{1}$ and $p_{1}$ respectively. The coefficient $\nu>0$ is the fluid kinematic viscosity, the function $\boldsymbol{f}_{1}$ is an external force acting on the fluid, and $\boldsymbol{D}\left(\boldsymbol{u}_{1}\right)$ is the rate of strain matrix:

$$
\boldsymbol{D}\left(\boldsymbol{u}_{1}\right)=\frac{1}{2}\left(\nabla \boldsymbol{u}_{1}+\nabla \boldsymbol{u}_{1}^{T}\right) .
$$

The flow in $\Omega_{2}$ is modeled by Darcy's Law:

$$
\begin{aligned}
-\nabla \cdot \boldsymbol{K} \nabla p_{2} & =f_{2}, \quad \text { in } \Omega_{2}, \\
-\boldsymbol{K} \nabla p_{2} & =\boldsymbol{u}_{2}, \quad \text { in } \Omega_{2}, \\
p_{2} & =g_{\mathrm{D}}, \quad \text { on } \Gamma_{2 \mathrm{D}}, \\
\boldsymbol{K} \nabla p_{2} \cdot \boldsymbol{n}_{2} & =g_{\mathrm{N}}, \quad \text { on } \Gamma_{2 \mathrm{~N}} .
\end{aligned}
$$

We note that the boundary of the porous medium $\Gamma_{2}=\partial \Omega \cap \partial \Omega_{2}$ is partitioned into disjoint sets $\Gamma_{2 \mathrm{~N}}$ and $\Gamma_{2 \mathrm{D}}$, the Neumann and Dirichlet boundary respectively. We assume that $\left|\Gamma_{2 \mathrm{D}}\right|>0$. The fluid velocity and pressure in $\Omega_{2}$ are denoted by $\boldsymbol{u}_{2}$ and $p_{2}$ respectively. The function $f_{2}$ is an external force acting on the fluid, the functions $g_{\mathrm{D}}$ and $g_{\mathrm{N}}$ are the prescribed value and flux respectively. The vector $\boldsymbol{n}_{2}$ denotes the unit vector normal to $\Gamma_{2}$ and the coefficient $\boldsymbol{K}$ is a symmetric positive definite tensor representing the hydraulic conductivity of the fluid.

The coupled models are completed by specifying the following coupling conditions on the interface $\Gamma_{12}$ :

(1) The continuity of the normal component of velocity arises from the incompressibility condition. Let $\boldsymbol{n}_{12}$ be the unit normal vector to $\Gamma_{12}$ directed from $\Omega_{1}$ to $\Omega_{2}$.

$$
\boldsymbol{u}_{1} \cdot \boldsymbol{n}_{12}=-\boldsymbol{K} \nabla p_{2} \cdot \boldsymbol{n}_{12}
$$

(2) The second condition is on the tangential component of the velocity in the free flow region. Let $\boldsymbol{\tau}_{12}$ be the unit tangent vector on the interface $\Gamma_{12}$ then the Beavers-Joseph-Saffman law $[13,14,15]$ will be written as:

$$
\boldsymbol{u}_{1} \cdot \boldsymbol{\tau}_{12}=-2 \nu G\left(\boldsymbol{D}\left(\boldsymbol{u}_{1}\right) \boldsymbol{n}_{12}\right) \cdot \boldsymbol{\tau}_{12}
$$

(3) The last condition relates to the balance of forces on the interface:

$$
-2 \nu \boldsymbol{D}\left(\boldsymbol{u}_{1}\right) \boldsymbol{n}_{12} \cdot \boldsymbol{n}_{12}+p_{1}=p_{2} .
$$




\section{$3 \quad$ Numerical Schemes}

Let $\mathcal{E}_{h}=\mathcal{E}_{1}^{h} \cup \mathcal{E}_{2}^{h}$ be a regular triangulation of the computational domain $\Omega$ with elements $E$ [16]. The velocity and pressure solutions on $\Omega_{1}$ belong to the conforming MINI finite element spaces $\boldsymbol{X}_{1}^{h}$ and $M_{1}^{h}$ respectively. We recall that the space $\boldsymbol{X}_{1}^{h}$ consists of continuous piecewise linears plus cubic bubbles and the space $M_{1}^{h}$ consists of continuous piecewise linears [17]. In $\Omega_{1}$, this yields a first order method. The Darcy pressure belongs to the space of discontinuous piecewise polynomials of degree $k_{2} \geq 1$ :

$$
M_{2}^{h}=\left\{q_{2} \in L^{2}\left(\Omega_{2}\right): \forall E \in \mathcal{E}_{2}^{h},\left.\quad q_{2}\right|_{E} \in \mathbb{P}_{k_{2}}(E)\right\} .
$$

We denote by $\Gamma_{2}^{h}$ the set of interior edges of $\mathcal{E}_{2}^{h}$. In addition, a unit normal vector $\boldsymbol{n}_{e}$ is associated with each edge $e$. In the case that $e$ lies on the boundary $\partial \Omega_{2}$, then $\boldsymbol{n}_{e}$ is taken to be the outward unit normal vector to $\partial \Omega_{2}$. For any two elements $E_{i}$ and $E_{j}$ (with $i<j$ ) of $\mathcal{E}_{2}^{h}$ that share a common edge $e$, we define the jump $[v]$ and average $\{v\}$ of a piecewise discontinuous polynomial $v$ by

$$
[v]=\left(\left.v\right|_{E_{i}}\right)-\left(\left.v\right|_{E_{j}}\right) \text {, and }\{v\}=\frac{1}{2}\left(\left.v\right|_{E_{i}}+\frac{1}{2}\left(\left.v\right|_{E_{j}}\right)\right.
$$

respectively. The choice of the coupling of the continuous finite element method (FEM) with the discontinuous Galerkin method is due to the fact that the continuous finite element method is adequate for the free flow region for problems considered in this work. On the other hand, the discontinuous Galerkin method is better suited to handle heterogeneities such as kinks and faults that arise in the porous medium. We refer the reader to [18] for a detailed discussion on this matter. In order to define the numerical scheme for the Stokes/Darcy and Navier-Stokes/Darcy couplings, we first introduce the following bilinear forms.

- For the discretization of the viscous term in the Stokes or Navier-Stokes equations:

$$
\forall \boldsymbol{v}, \boldsymbol{w} \in \boldsymbol{X}_{1}^{h}, \quad a_{\mathrm{S}}(\boldsymbol{v}, \boldsymbol{w})=2 \nu(\boldsymbol{D}(\boldsymbol{v}), \boldsymbol{D}(\boldsymbol{w}))_{\Omega_{1}}
$$

- For the discretization of the pressure term in the Stokes or Navier-Stokes equations:

$$
\forall \boldsymbol{v} \in \boldsymbol{X}_{1}^{h}, \forall q_{1} \in M_{1}^{h}, \quad b_{\mathrm{S}}\left(\boldsymbol{v}, q_{1}\right)=-\left(q_{1}, \nabla \cdot \boldsymbol{v}\right)_{\Omega_{1}} .
$$

- For the discretization of the non-linear term in the Navier-Stokes equations:

$$
\begin{gathered}
\forall \boldsymbol{z}, \boldsymbol{v}, \boldsymbol{w} \in \boldsymbol{X}_{1}^{h} \\
c_{\mathrm{NS}}(\boldsymbol{z}, \boldsymbol{v}, \boldsymbol{w})=\frac{1}{2}(\boldsymbol{z} \cdot \nabla \boldsymbol{v}, \boldsymbol{w})_{\Omega_{1}}-\frac{1}{2}(\boldsymbol{z} \cdot \nabla \boldsymbol{w}, \boldsymbol{v})_{\Omega_{1}}+\frac{1}{2}\left(\boldsymbol{z} \cdot \boldsymbol{n}_{12}, \boldsymbol{v} \cdot \boldsymbol{w}\right)_{\Gamma_{12}}
\end{gathered}
$$


- For the discretization of the diffusion term in Darcy's Law:

$$
\begin{gathered}
\forall q_{2}, t_{2} \in M_{2}^{h}, \quad a_{\mathrm{D}}\left(q_{2}, t_{2}\right)=\sum_{E \in \mathcal{E}_{h}^{2}}\left(\boldsymbol{K} \nabla q_{2}, \nabla t_{2}\right)_{E}+\sum_{e \in \Gamma_{2}^{h} \cup \Gamma_{2 \mathrm{D}}} \frac{\sigma}{|e|}\left(\left[q_{2}\right],\left[t_{2}\right]\right)_{e} \\
-\sum_{e \in \Gamma_{2}^{h} \cup \Gamma_{2 \mathrm{D}}}\left(\left\{\boldsymbol{K} \nabla q_{2} \cdot \boldsymbol{n}_{e}\right\}\left[t_{2}\right]\right)_{e}+\epsilon_{2} \sum_{e \in \Gamma_{2}^{h} \cup \Gamma_{2 \mathrm{D}}}\left(\left\{\boldsymbol{K} \nabla t_{2} \cdot \boldsymbol{n}_{e}\right\},\left[q_{2}\right]\right)_{e} .
\end{gathered}
$$

The parameters $\sigma$ and $\epsilon_{2}$ are the penalty and symmetrization parameters respectively. The case $\epsilon_{2}=-1$ yields the Symmetric Interior Penalty Galerkin (SIPG) method whereas the case $\epsilon_{2}=1$ yields the Non-Symmetric Interior Penalty Galerkin (NIPG) method [19]. We also note that the form $a_{\mathrm{D}}$ depends strongly on the underlying mesh. We may use the notation $a_{\mathrm{D}}=a_{\mathrm{D}, h}$ in the definition of the two-grid method to avoid any confusion.

- For the discretization of the interface terms:

$$
\begin{gathered}
\forall \boldsymbol{v}, \boldsymbol{w} \in \boldsymbol{X}_{1}^{h}, \quad \forall q_{2}, t_{2} \in M_{2}^{h}, \quad \gamma\left(\boldsymbol{v}, q_{2} ; \boldsymbol{w}, t_{2}\right)=\left(q_{2}, \boldsymbol{w} \cdot \boldsymbol{n}_{12}\right)_{\Gamma_{12}}-\left(\boldsymbol{v} \cdot \boldsymbol{n}_{12}, t_{2}\right)_{\Gamma_{12}} \\
+\frac{1}{G}\left(\boldsymbol{v} \cdot \boldsymbol{\tau}_{12}, \boldsymbol{w} \cdot \boldsymbol{\tau}_{12}\right)_{\Gamma_{12}} .
\end{gathered}
$$

- For the discretization of the data terms:

$$
\begin{gathered}
\forall t_{2} \in M_{2}^{h}, \forall \boldsymbol{v} \in \boldsymbol{X}_{1}^{h}, \quad L\left(\boldsymbol{v}, t_{2}\right)=\left(\boldsymbol{f}_{1}, \boldsymbol{v}\right)_{\Omega_{1}}+\left(f_{2}, t_{2}\right)_{\Omega_{2}} \\
+\epsilon_{2} \sum_{e \in \Gamma_{2 \mathrm{D}}}\left(\boldsymbol{K} \nabla t_{2} \cdot \boldsymbol{n}_{e}, g_{\mathrm{D}}\right)_{e}+\sum_{e \in \Gamma_{2 \mathrm{D}}} \frac{\sigma}{|e|}\left(g_{\mathrm{D}}, t_{2}\right)_{e}+\sum_{e \in \Gamma_{2 \mathrm{~N}}}\left(g_{\mathrm{N}}, t_{2}\right)_{e} .
\end{gathered}
$$

We now define the numerical schemes for the coupled Stokes/Darcy and coupled Navier-Stokes/Darcy problems $\left(W_{\mathrm{S}}^{h}\right)$ and $\left(W_{\mathrm{NS}}^{h}\right)$ respectively:

$$
\begin{gathered}
\left(W_{\mathrm{S}}^{h}\right)\left\{\begin{array}{l}
\text { Find } \boldsymbol{U}_{1}^{h} \in \boldsymbol{X}_{1}^{h}, P_{1}^{h} \in M_{1}^{h}, P_{2}^{h} \in M_{2}^{h}, \text { s.t } \\
\forall \boldsymbol{v}_{1} \in \boldsymbol{X}_{1}^{h}, q_{2} \in M_{2}^{h}, a_{\mathrm{S}}\left(\boldsymbol{U}_{1}^{h}, \boldsymbol{v}_{1}\right)+b_{\mathrm{S}}\left(\boldsymbol{v}_{1}, P_{1}^{h}\right)+a_{\mathrm{D}}\left(P_{2}^{h}, q_{2}\right) \\
+\gamma\left(\boldsymbol{U}_{1}^{h}, P_{2}^{h} ; \boldsymbol{v}_{1}, q_{2}\right)=L\left(\boldsymbol{v}_{1}, q_{2}\right), \\
\forall q_{1} \in M_{1}^{h} \quad b_{\mathrm{S}}\left(\boldsymbol{U}_{1}^{h}, q_{1}\right)=0,
\end{array}\right. \\
\left(W_{\mathrm{NS}}^{h}\right)\left\{\begin{array}{l}
\text { Find } \boldsymbol{U}_{1}^{h} \in \boldsymbol{X}_{1}^{h}, P_{1}^{h} \in M_{1}^{h}, P_{2}^{h} \in M_{2}^{h}, \text { s.t } \\
\forall \boldsymbol{v}_{1} \in \boldsymbol{X}_{1}^{h}, q_{2} \in M_{2}^{h}, a_{\mathrm{S}}\left(\boldsymbol{U}_{1}^{h}, \boldsymbol{v}_{1}\right)+b_{\mathrm{S}}\left(\boldsymbol{v}_{1}, P_{1}^{h}\right)+c_{\mathrm{NS}}\left(\boldsymbol{U}_{1}^{h} ; \boldsymbol{U}_{1}^{h}, \boldsymbol{v}_{1}\right) \\
+a_{\mathrm{D}}\left(P_{2}^{h}, q_{2}\right)+\gamma\left(\boldsymbol{U}_{1}^{h}, P_{2}^{h} ; \boldsymbol{v}_{1}, q_{2}\right)=L\left(\boldsymbol{v}_{1}, q_{2}\right), \\
\forall q_{1} \in M_{1}^{h} \quad b_{\mathrm{S}}\left(\boldsymbol{U}_{1}^{h}, q_{1}\right)=0 .
\end{array}\right.
\end{gathered}
$$

In order to solve the coupled problem $\left(W_{\mathrm{S}}^{h}\right)$, we have to assemble a large linear system of dimension $\operatorname{dim} \boldsymbol{X}_{1}^{h}+\operatorname{dim} M_{1}^{h}+\operatorname{dim} M_{2}^{h}$. For realistic domains 
this system can be very large and time consuming to solve. Similarly, solving $\left(W_{\mathrm{NS}}^{h}\right)$ is time consuming as it involves a Picard iteration and large linear systems. In the next section we present a two-grid algorithm that reduces the computational cost by decoupling the coupled problems into two relatively smaller problems in the free flow and porous media domains.

\section{Two Grid Algorithm}

The objective of the two-grid method is to decouple the fully coupled problem into separate problems in the free flow and porous media domains. The technique involves two steps. First, a fully coupled problem, with relatively small cost, is solved on a coarse mesh $\mathcal{E}_{H}$ of size $H$. Second, the surface problem and subsurface problems are solved in parallel and independently on a fine mesh $\mathcal{E}_{h}$ of size $h$. The two-grid algorithm is summarized below for both linear and nonlinear cases.

\subsection{Two-grid algorithm for coupled Stokes/Darcy problem}

(1) First we solve a fully coupled Stokes and Darcy problem on a coarse grid of size $H$ :

$$
\left(W_{\mathrm{S}}^{H}\right)\left\{\begin{array}{l}
\text { Find } \boldsymbol{U}_{1}^{H} \in \boldsymbol{X}_{1}^{H}, P_{1}^{H} \in M_{1}^{H}, P_{2}^{H} \in M_{2}^{H}, \text { s.t. } \\
\forall \boldsymbol{v}_{1} \in \boldsymbol{X}_{1}^{H}, \forall q_{2} \in M_{2}^{H}, a_{\mathrm{S}}\left(\boldsymbol{U}_{1}^{H}, \boldsymbol{v}_{1}\right)+b_{\mathrm{S}}\left(\boldsymbol{v}_{1}, P_{1}^{H}\right)+a_{\mathrm{D}, H}\left(P_{2}^{H}, q_{2}\right) \\
+\gamma\left(\boldsymbol{U}_{1}^{H}, P_{2}^{H} ; \boldsymbol{v}_{1}, q_{2}\right)=L\left(\boldsymbol{v}_{1}, q_{2}\right) \\
\forall q_{1} \in M_{1}^{H}, \quad b_{\mathrm{S}}\left(\boldsymbol{U}_{1}^{H}, q_{1}\right)=0 .
\end{array}\right.
$$

(2) We then solve a decoupled problem on a fine grid of size $h$ :

$$
\left(W_{h, \mathrm{SD}}\right)\left\{\begin{array}{l}
\text { Find } \boldsymbol{U}_{h, 1} \in \boldsymbol{X}_{1}^{h}, P_{h, 1} \in M_{1}^{h}, P_{h, 2} \in M_{2}^{h}, \text { s.t. } \\
\forall \boldsymbol{v}_{1} \in \boldsymbol{X}_{1}^{h}, \forall q_{2} \in M_{2}^{h}, a_{\mathrm{S}}\left(\boldsymbol{U}_{h, 1}, \boldsymbol{v}_{1}\right)+\frac{1}{G}\left(\boldsymbol{U}_{h, 1} \cdot \boldsymbol{\tau}_{12}, \boldsymbol{v}_{1} \cdot \boldsymbol{\tau}_{12}\right)_{\Gamma_{12}}+b_{\mathrm{S}}\left(\boldsymbol{v}_{1}, P_{h, 1}\right) \\
+a_{\mathrm{D}, h}\left(P_{h, 2}, q_{2}\right)=L\left(\boldsymbol{v}_{1}, q_{2}\right)-\left(P_{2}^{H}, \boldsymbol{v}_{1} \cdot \boldsymbol{n}_{12}\right)_{\Gamma_{12}}+\left(\boldsymbol{U}_{1}^{H} \cdot \boldsymbol{n}_{12}, q_{2}\right)_{\Gamma_{12}}, \\
\forall q_{1} \in M_{1}^{h}, \quad b_{\mathrm{S}}\left(\boldsymbol{U}_{h, 1}, q_{1}\right)=0 .
\end{array}\right.
$$

The decoupled fine grid problem is equivalent to solving modified Stokes and Darcy problems in $\Omega_{1}$ and $\Omega_{2}$ respectively. The modified problems can be 
stated as follows: in $\Omega_{1}$,

$\left(W_{h, \mathrm{~S}}\right)\left\{\begin{array}{l}\text { Find } \boldsymbol{U}_{h, 1} \in \boldsymbol{X}_{1}^{h}, P_{h, 1} \in M_{1}^{h}, \text { s.t. } \\ \forall \boldsymbol{v}_{1} \in \boldsymbol{X}_{1}^{h}, \forall q_{2} \in M_{2}^{h}, a_{\mathrm{S}}\left(\boldsymbol{U}_{h, 1}, \boldsymbol{v}_{1}\right)+b_{\mathrm{S}}\left(\boldsymbol{v}_{1}, P_{h, 1}\right)+\frac{1}{G}\left(\boldsymbol{U}_{h, 1} \cdot \boldsymbol{\tau}_{12}, \boldsymbol{v}_{1} \cdot \boldsymbol{\tau}_{12}\right)_{\Gamma_{12}} \\ =\left(\boldsymbol{f}_{1}, \boldsymbol{v}_{1}\right)_{\Omega_{1}}-\left(P_{2}^{H}, \boldsymbol{v}_{1} \cdot \boldsymbol{n}_{12}\right)_{\Gamma_{12}}, \\ \forall q_{1} \in M_{1}^{h}, \quad b_{\mathrm{S}}\left(\boldsymbol{U}_{h, 1}, q_{1}\right)=0,\end{array}\right.$

and in $\Omega_{2}$,

$\left(W_{h, \mathrm{D}}\right)\left\{\begin{array}{l}\text { Find } P_{h, 2} \in M_{2}^{h}, \text { s.t. } \\ \forall q_{2} \in M_{2}^{h}, \quad a_{\mathrm{D}, h}\left(P_{h, 2}, q_{2}\right)=\left(f_{2}, q_{2}\right)_{\Omega_{2}}+\left(\boldsymbol{U}_{1}^{H} \cdot \boldsymbol{n}_{12}, q_{2}\right)_{\Gamma_{12}}+\left(g_{\mathrm{N}}, q_{2}\right)_{\Gamma_{2 \mathrm{~N}}} \\ +\epsilon_{2} \sum_{e \in \Gamma_{2 \mathrm{D}}}\left(\boldsymbol{K} \nabla q_{2} \cdot \boldsymbol{n}_{e}, g_{\mathrm{D}}\right)_{e}+\sum_{e \in \Gamma_{2 \mathrm{D}}} \frac{\sigma}{|e|}\left(g_{\mathrm{D}}, q_{2}\right)_{e} .\end{array}\right.$

4.2 Two-grid algorithm for coupled Navier-Stokes and Darcy problem

(1) First we solve a nonlinear problem on a coarse grid of size $H$ :

$\left(W_{\mathrm{NS}}^{H}\right)\left\{\begin{array}{l}\text { Find } \boldsymbol{U}_{1}^{H} \in \boldsymbol{X}_{1}^{h}, P_{1}^{H} \in M_{1}^{H}, P_{2}^{H} \in M_{2}^{H}, \text { s.t. } \\ \forall \boldsymbol{v}_{1} \in \boldsymbol{X}_{1}^{H}, \forall q_{2} \in M_{2}^{H}, a_{\mathrm{S}}\left(\boldsymbol{U}_{1}^{H}, \boldsymbol{v}_{1}\right)+b_{\mathrm{S}}\left(\boldsymbol{v}_{1}, P_{1}^{H}\right)+c_{\mathrm{NS}}\left(\boldsymbol{U}_{1}^{H} ; \boldsymbol{U}_{1}^{H} ; \boldsymbol{v}_{1}\right) \\ +a_{\mathrm{D}, H}\left(P_{2}^{H}, q_{2}\right)+\gamma\left(\boldsymbol{U}_{1}^{H}, P_{2}^{H} ; \boldsymbol{v}_{1}, q_{2}\right)=L\left(\boldsymbol{v}_{1}, q_{2}\right), \\ \forall q_{1} \in M_{1}^{H}, b_{\mathrm{S}}\left(\boldsymbol{U}_{1}^{H}, q_{1}\right)=0 .\end{array}\right.$

The solution $\left(\boldsymbol{U}_{1}^{H}, P_{1}^{H}, P_{2}^{H}\right)$ for the problem $\left(W_{\mathrm{NS}}^{H}\right)$ is obtained using a $\mathrm{Pi}$ card iteration. At each iteration, given $\boldsymbol{U}_{1}^{H, n-1}$, we solve for $\left(\boldsymbol{U}_{1}^{H, n}, P_{1}^{H}, P_{2}^{H}\right)$ satisfying:

$$
\begin{gathered}
\forall \boldsymbol{v}_{1} \in \boldsymbol{X}_{1}^{H}, \forall q_{2} \in M_{2}^{H}, \quad a_{\mathrm{S}}\left(\boldsymbol{U}_{1}^{H, n}, \boldsymbol{v}_{1}\right)+b_{\mathrm{S}}\left(\boldsymbol{v}_{1}, P_{1}^{H}\right)+c_{\mathrm{NS}}\left(\boldsymbol{U}_{1}^{H, n-1} ; \boldsymbol{U}_{1}^{H, n} ; \boldsymbol{v}_{1}\right) \\
+a_{\mathrm{D}, H}\left(P_{2}^{H}, q_{2}\right)+\gamma\left(\boldsymbol{U}_{1}^{H, n}, P_{2}^{H} ; \boldsymbol{v}_{1}, q_{2}\right)=L\left(\boldsymbol{v}_{1}, q_{2}\right), \\
\forall q_{1} \in M_{1}^{H}, \quad b_{\mathrm{S}}\left(\boldsymbol{U}_{1}^{H, n}, q_{1}\right)=0 .
\end{gathered}
$$

We use the stopping criterion: $\left\|\boldsymbol{U}_{1}^{H, n}-\boldsymbol{U}_{1}^{H, n-1}\right\|_{L^{2}\left(\Omega_{1}\right)} \leq \delta$, where $\delta$ is a given tolerance. 
(2) We then solve a decoupled problem on a fine grid of size $h$ :

$$
\left(W_{h, \mathrm{NSD}}\right)\left\{\begin{array}{l}
\text { Find } \boldsymbol{U}_{h, 1} \in \boldsymbol{X}_{1}^{h}, P_{h, 1} \in M_{1}^{h}, P_{h, 2} \in M_{2}^{h}, \text { s.t. } \\
\forall \boldsymbol{v}_{1} \in \boldsymbol{X}_{1}^{h}, \forall q_{2} \in M_{2}^{h}, \quad a_{\mathrm{S}}\left(\boldsymbol{U}_{h, 1}, \boldsymbol{v}_{1}\right)+b_{\mathrm{S}}\left(\boldsymbol{v}_{1}, P_{h, 1}\right)+c_{\mathrm{NS}}\left(\boldsymbol{U}_{1}^{H} ; \boldsymbol{U}_{h, 1}, \boldsymbol{v}_{1}\right) \\
+\frac{1}{G}\left(\boldsymbol{U}_{h, 1} \cdot \boldsymbol{\tau}_{12}, \boldsymbol{v}_{1} \cdot \boldsymbol{\tau}_{12}\right)_{\Gamma_{12}}+a_{\mathrm{D}, h}\left(P_{h, 2}, q_{2}\right) \\
=L\left(\boldsymbol{v}_{1}, q_{2}\right)-\left(P_{2}^{H}, \boldsymbol{v}_{1} \cdot \boldsymbol{n}\right)_{\Gamma_{12}}+\left(\boldsymbol{U}_{1}^{H} \cdot \boldsymbol{n}_{12}, q_{2}\right)_{\Gamma_{12}}, \\
\forall q_{1} \in M_{1}^{h}, \quad b_{\mathrm{S}}\left(\boldsymbol{U}_{h, 1}, q_{1}\right)=0 .
\end{array}\right.
$$

We note that the resulting two-grid problem $\left(W_{h, \mathrm{NSD}}\right)$ does not require a Picard iteration because the non-linear term $c_{\mathrm{NS}}$ has been linearized. The decoupled fine grid problem is equivalent to solving modified Oseen and Darcy problems in $\Omega_{1}$ and $\Omega_{2}$ respectively. The modified problems can be stated as follows: in $\Omega_{1}$,

$$
\left(W_{h, \mathrm{NS}}\right)\left\{\begin{array}{l}
\text { Find } \boldsymbol{U}_{h, 1} \in \boldsymbol{X}_{1}^{h}, P_{h, 1} \in M_{1}^{h}, \text { s.t. } \\
\forall \boldsymbol{v}_{1} \in \boldsymbol{X}_{1}^{h}, \forall q_{2} \in M_{2}^{h}, a_{\mathrm{S}}\left(\boldsymbol{U}_{h, 1}, \boldsymbol{v}_{1}\right)+b_{\mathrm{S}}\left(\boldsymbol{v}_{1}, P_{h, 1}\right)+c_{\mathrm{NS}}\left(\boldsymbol{U}_{1}^{H} ; \boldsymbol{U}_{h, 1}, \boldsymbol{v}_{1}\right) \\
+\frac{1}{G}\left(\boldsymbol{U}_{h, 1} \cdot \boldsymbol{\tau}_{12}, \boldsymbol{v}_{1} \cdot \boldsymbol{\tau}_{12}\right)_{\Gamma_{12}}=\left(\boldsymbol{f}_{1}, \boldsymbol{v}_{1}\right)_{\Omega_{1}}-\left(P_{2}^{H}, \boldsymbol{v}_{1} \cdot \boldsymbol{n}\right)_{\Gamma_{12}}, \\
\forall q_{1} \in M_{1}^{h}, \quad b_{\mathrm{S}}\left(\boldsymbol{U}_{h, 1}, q_{1}\right)=0,
\end{array}\right.
$$

and in $\Omega_{2}$,

$$
\left(W_{h, \mathrm{D}}\right)\left\{\begin{array}{l}
\text { Find } P_{h, 2} \in M_{2}^{h}, \text { s.t. } \\
\forall q_{2} \in M_{2}^{h}, \quad a_{\mathrm{D}, h}\left(P_{h, 2}, q_{2}\right)=\left(f_{2}, q_{2}\right)_{\Omega_{2}}+\left(\boldsymbol{U}_{1}^{H} \cdot \boldsymbol{n}_{12}, q_{2}\right)_{\Gamma_{12}}+\left(g_{\mathrm{N}}, q_{2}\right)_{\Gamma_{2 \mathrm{~N}}} \\
+\epsilon_{2} \sum_{e \in \Gamma_{2 \mathrm{D}}}\left(\boldsymbol{K} \nabla q_{2} \cdot \boldsymbol{n}_{e}, g_{\mathrm{D}}\right)_{e}+\sum_{e \in \Gamma_{2 \mathrm{D}}} \frac{\sigma}{|e|}\left(g_{\mathrm{D}}, q_{2}\right)_{e} .
\end{array}\right.
$$

Remark 1 For all schemes described above, the Darcy velocity is obtained by the following formula:

$$
\boldsymbol{U}_{h, 2}=-\boldsymbol{K} \nabla P_{h, 2}
$$

\subsection{Convergence Result}

Under the assumption that the solution to the dual coupled problem is regular enough, one can show that there exists a constant $C$ independent of $H$ and $h$ such that 


$$
\begin{array}{r}
\left(\sum_{E \in \mathcal{E}_{2}^{h}}\left\|\nabla\left(P_{2}^{h}-P_{h, 2}\right)\right\|_{L^{2}(E)}^{2}+\sum_{e \in \Gamma_{h}^{2}} \frac{1}{|e|}\left\|\left[P_{2}^{h}-P_{h, 2}\right]\right\|_{L^{2}(e)}^{2}\right)^{1 / 2} \leq C H^{2} \\
\left\|\boldsymbol{D}\left(\boldsymbol{U}_{1}^{h}-\boldsymbol{U}_{h, 1}\right)\right\|_{L^{2}\left(\Omega_{1}\right)}+\left\|P_{1}^{h}-P_{h, 1}\right\|_{L^{2}\left(\Omega_{1}\right)} \leq C H^{3 / 2} .
\end{array}
$$

Therefore, if one chooses $h=H^{2}$, we obtain an overall theoretical rate $\mathcal{O}\left(h^{3 / 4}\right)$. In the following section, we study the numerical convergence and we observe improved numerical rates $\mathcal{O}(h)$.

\section{$5 \quad$ Numerical Examples}

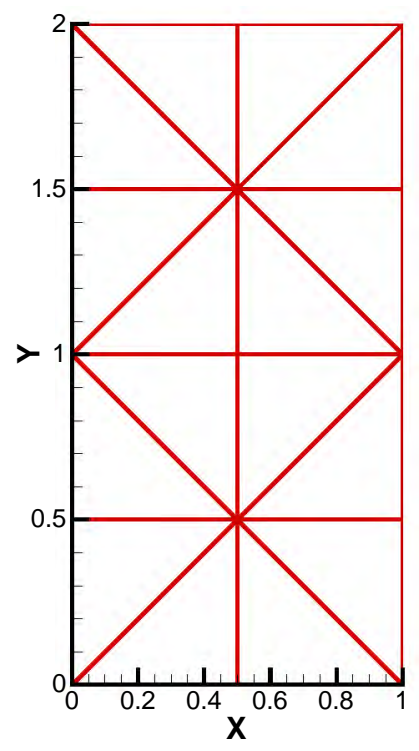

(a) Coarse Mesh $\mathcal{E}_{H}$

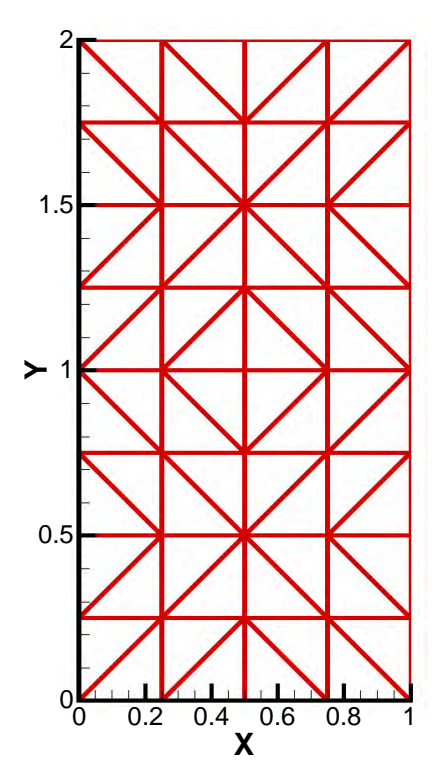

(b) Fine Mesh $\mathcal{E}_{h}$

Fig. 1. Computational meshes $H=0.5, h=0.25$.

We compute numerical convergence rates for the two-grid algorithm applied to both the coupled Stokes/Darcy and coupled Navier-Stokes/Darcy problems. We also compare the accuracy of the two-grid solution with the solution obtained by solving the fully coupled problem. The computational domain $\Omega \subset \mathbb{R}^{2}$ is subdivided into $\Omega_{1}=(0,1) \times(0,1)$ and $\Omega_{2}=(0,1) \times(1,2)$. For each given mesh of size $h$, we solve the fully coupled problem on a coarse mesh of size $H=\sqrt{h}$ and the decoupled problems on a fine mesh of size $h$. An example of such meshes is given in Fig. 1. The boundary conditions are chosen in such a way that the exact solution to the coupled problem for both models is:

$$
\begin{gathered}
\boldsymbol{u}_{1}(x, y)=\left(y^{2}-2 y+2 x, x^{2}-x-2 y\right), \quad p_{1}(x, y)=x^{2} y+x y+y^{2}-4.0 \\
p_{2}(x, y)=-x^{2} y+x y+y^{2} .
\end{gathered}
$$


For simplicity we set $\boldsymbol{K}=\boldsymbol{I}$ and $\nu=1$.

\subsection{Convergence Study for Decoupled Stokes and Darcy problem}

In this section we compare the accuracy of the two-grid method for the coupled Stokes and Darcy model. First we solve the fully coupled problem on a sequence of fine meshes of size $h$. For the subsurface part, the NIPG method is used with penalty $\sigma=1$ and with order of approximation $k_{2}=1$. Table 1 shows the errors and convergence rates for the fully coupled problem. As expected from the theory [4] we observe order one convergence rate in the Stokes and Darcy velocities in the energy norm. The Stokes pressure has a slightly higher convergence rate than the expected order one. We also show the rates for the Stokes velocity and Darcy pressure in the $L^{2}$ norm. We recover the optimal rate of two.

\begin{tabular}{|c|c|c|c|c|c|}
\hline$h$ & $\left\|\boldsymbol{U}_{1}^{h}-\boldsymbol{u}_{1}\right\|_{L^{2}\left(\Omega_{1}\right)}$ & $\left\|P_{1}^{h}-p_{1}\right\|_{L^{2}\left(\Omega_{1}\right)}$ & $\left\|\boldsymbol{D}\left(\boldsymbol{U}_{1}^{h}-\boldsymbol{u}_{1}\right)\right\|_{L^{2}\left(\Omega_{1}\right)}$ & $\left\|P_{2}^{h}-p_{2}\right\|_{L^{2}\left(\Omega_{2}\right)}$ & $\left\|\nabla\left(P_{2}^{h}-p_{2}\right)\right\|_{L^{2}\left(\Omega_{2}\right)}$ \\
\hline$\frac{1}{4}$ & $1.591 \times 10^{-2}$ & $6.346 \times 10^{-2}$ & $1.365 \times 10^{-1}$ & $1.341 \times 10^{-2}$ & $1.637 \times 10^{-1}$ \\
$\frac{1}{16}$ & $9.984 \times 10^{-4}$ & $6.645 \times 10^{-3}$ & $3.390 \times 10^{-2}$ & $8.210 \times 10^{-4}$ & $3.856 \times 10^{-2}$ \\
$\frac{1}{64}$ & $6.227 \times 10^{-5}$ & $7.363 \times 10^{-4}$ & $8.469 \times 10^{-3}$ & $5.150 \times 10^{-5}$ & $9.552 \times 10^{-3}$ \\
\hline rate $(h)$ & 2.00 & 1.58 & 1.00 & 2.00 & 1.00 \\
\hline
\end{tabular}

Table 1

Errors and rates for $\left(W_{\mathrm{S}}^{h}\right)\left(\mathrm{FEM} / \mathrm{NIPG} \sigma=1, k_{2}=1\right)$.

We then solve problem $\left(W_{h, \mathrm{SD}}\right)$ on the same sequence of meshes as in Table 1. Tables 2 and 3 show the errors and convergence rates for $\left(W_{h, \mathrm{SD}}\right)$ using the NIPG method with $\sigma=1$ and the SIPG method with $\sigma=6$ in $\Omega_{2}$ respectively. First we note that the errors obtained from the NIPG and SIPG solutions are comparable. For the SIPG method the expected convergence rate is obtained by making the penalty parameter large enough. This is due to the fact that the coercivity of the bilinear form $a_{\mathrm{D}}$ for the SIPG method depends on the choice of the penalty parameter. From [20], we choose $\sigma=k_{2}\left(k_{2}+1\right) \cot (\theta)$, where $\theta$ is the smallest angle over all triangles in the mesh. We obtain order one convergence for the errors in the energy norm in the parameter $h$ for both methods. The order of convergence is better than the predicted theoretical convergence rate of $h^{3 / 4}$. The errors are comparable to those obtained by solving the fully coupled problem (Table 1) for both methods. However the Stokes pressure error is relatively larger for the two-grid problem compared to the fully coupled problem.

Next we increase the degree of approximation in $\Omega_{2}$ to two. Table 4 and 5 show the errors and convergence rates for the two grid algorithm using the NIPG method with $\sigma=0$ and SIPG method with $\sigma=18$ in $\Omega_{2}$ respectively. Increasing the degree of approximation in $\Omega_{2}$ improves the quality of the Darcy pressure solution. For example on a mesh of size $\frac{1}{64}$ the error in the Darcy 


\begin{tabular}{|c|c|c|c|c|c|c|}
\hline$h$ & $H$ & $\left\|\boldsymbol{U}_{h, 1}-\boldsymbol{u}_{1}\right\|_{L^{2}\left(\Omega_{1}\right)}$ & $\left\|P_{h, 1}-p_{1}\right\|_{L^{2}\left(\Omega_{1}\right)}$ & $\left\|\boldsymbol{D}\left(\boldsymbol{U}_{h, 1}-\boldsymbol{u}_{1}\right)\right\|_{L^{2}\left(\Omega_{1}\right)}$ & $\left\|P_{h, 2}-p_{2}\right\|_{L^{2}\left(\Omega_{2}\right)}$ & $\left\|\nabla\left(P_{h, 2}-p_{2}\right)\right\|_{L^{2}\left(\Omega_{2}\right)}$ \\
\hline$\frac{1}{4}$ & $\frac{1}{2}$ & $1.599 \times 10^{-2}$ & $6.647 \times 10^{-2}$ & $1.364 \times 10^{-1}$ & $1.235 \times 10^{-2}$ & $1.657 \times 10^{-1}$ \\
$\frac{1}{16}$ & $\frac{1}{4}$ & $9.984 \times 10^{-4}$ & $1.571 \times 10^{-2}$ & $3.397 \times 10^{-2}$ & $1.018 \times 10^{-3}$ & $3.923 \times 10^{-2}$ \\
$\frac{1}{64}$ & $\frac{1}{8}$ & $6.073 \times 10^{-5}$ & $4.349 \times 10^{-3}$ & $8.473 \times 10^{-3}$ & $2.699 \times 10^{-4}$ & $9.638 \times 10^{-3}$ \\
\hline rate $(h)$ & - & 2.00 & 0.92 & 1.00 & 0.95 & 1.00 \\
\hline
\end{tabular}

Table 2

Errors and rates for $\left(W_{h, \mathrm{SD}}\right)\left(\mathrm{FEM} / \mathrm{NIPG} \sigma=1, k_{2}=1\right)$.

\begin{tabular}{|c|c|c|c|c|c|c|}
\hline$h$ & $H$ & $\left\|\boldsymbol{U}_{h, 1}-\boldsymbol{u}_{1}\right\|_{L^{2}\left(\Omega_{1}\right)}$ & $\left\|P_{h, 1}-p_{1}\right\|_{L^{2}\left(\Omega_{1}\right)}$ & $\left\|\boldsymbol{D}\left(\boldsymbol{U}_{h, 1}-\boldsymbol{u}_{1}\right)\right\|_{L^{2}\left(\Omega_{1}\right)}$ & $\left\|P_{h, 2}-p_{2}\right\|_{L^{2}\left(\Omega_{2}\right)}$ & $\left\|\nabla\left(P_{h, 2}-p_{2}\right)\right\|_{L^{2}\left(\Omega_{2}\right)}$ \\
\hline$\frac{1}{4}$ & $\frac{1}{2}$ & $1.594 \times 10^{-2}$ & $7.383 \times 10^{-2}$ & $1.363 \times 10^{-1}$ & $8.788 \times 10^{-3}$ & $1.703 \times 10^{-1}$ \\
$\frac{1}{16}$ & $\frac{1}{4}$ & $1.002 \times 10^{-3}$ & $1.489 \times 10^{-2}$ & $3.397 \times 10^{-2}$ & $1.471 \times 10^{-3}$ & $4.137 \times 10^{-2}$ \\
$\frac{1}{64}$ & $\frac{1}{8}$ & $6.616 \times 10^{-5}$ & $4.166 \times 10^{-3}$ & $8.473 \times 10^{-3}$ & $3.115 \times 10^{-4}$ & $1.011 \times 10^{-2}$ \\
\hline rate $(h)$ & - & 2.00 & 0.92 & 1.00 & 1.12 & 1.00 \\
\hline
\end{tabular}

Table 3

Errors and rates for $\left(W_{h, \mathrm{SD}}\right)\left(\mathrm{FEM} / \mathrm{SIPG} \sigma=6, k_{2}=1\right)$.

velocity in Table 4 is about seven times smaller compared to Tables 2 and 3. The Stokes velocity error is unchanged from the case $k_{2}=1$ to $k_{2}=2$. The Stokes pressure error decreases by a factor of four.

\begin{tabular}{|c|c|c|c|c|c|c|}
\hline$h$ & $H$ & $\left\|\boldsymbol{U}_{h, 1}-\boldsymbol{u}_{1}\right\|_{L^{2}\left(\Omega_{1}\right)}$ & $\left\|P_{h, 1}-p_{1}\right\|_{L^{2}\left(\Omega_{1}\right)}$ & $\left\|\boldsymbol{D}\left(\boldsymbol{U}_{h, 1}-\boldsymbol{u}_{1}\right)\right\|_{L^{2}\left(\Omega_{1}\right)}$ & $\left\|P_{h, 2}-p_{2}\right\|_{L^{2}\left(\Omega_{2}\right)}$ & $\left\|\nabla\left(P_{h, 2}-p_{2}\right)\right\|_{L^{2}\left(\Omega_{2}\right)}$ \\
\hline$\frac{1}{4}$ & $\frac{1}{2}$ & $1.599 \times 10^{-2}$ & $6.577 \times 10^{-2}$ & $1.363 \times 10^{-1}$ & $5.134 \times 10^{-3}$ & $2.556 \times 10^{-2}$ \\
$\frac{1}{16}$ & $\frac{1}{4}$ & $9.928 \times 10^{-4}$ & $7.708 \times 10^{-3}$ & $3.396 \times 10^{-2}$ & $1.213 \times 10^{-3}$ & $5.654 \times 10^{-3}$ \\
$\frac{1}{64}$ & $\frac{1}{8}$ & $6.258 \times 10^{-5}$ & $1.304 \times 10^{-3}$ & $8.470 \times 10^{-3}$ & $2.981 \times 10^{-4}$ & $1.378 \times 10^{-3}$ \\
\hline rate $(h)$ & - & 2.00 & 1.28 & 1.00 & 1.00 & 1.00 \\
\hline
\end{tabular}

Table 4

Errors and rates for $\left(W_{h, \mathrm{SD}}\right)\left(\mathrm{FEM} / \mathrm{NIPG} \sigma=0, k_{2}=2\right)$.

\begin{tabular}{|c|c|c|c|c|c|c|}
\hline$h$ & $H$ & $\left\|\boldsymbol{U}_{h, 1}-\boldsymbol{u}_{1}\right\|_{L^{2}\left(\Omega_{1}\right)}$ & $\left\|P_{h, 1}-p_{1}\right\|_{L^{2}\left(\Omega_{1}\right)}$ & $\left\|\boldsymbol{D}\left(\boldsymbol{U}_{h, 1}-\boldsymbol{u}_{1}\right)\right\|_{L^{2}\left(\Omega_{1}\right)}$ & $\left\|P_{h, 2}-p_{2}\right\|_{L^{2}\left(\Omega_{2}\right)}$ & $\left\|\nabla\left(P_{h, 2}-p_{2}\right)\right\|_{L^{2}\left(\Omega_{2}\right)}$ \\
\hline$\frac{1}{4}$ & $\frac{1}{2}$ & $1.599 \times 10^{-2}$ & $6.470 \times 10^{-2}$ & $1.364 \times 10^{-1}$ & $4.965 \times 10^{-3}$ & $2.470 \times 10^{-2}$ \\
$\frac{1}{16}$ & $\frac{1}{4}$ & $9.927 \times 10^{-4}$ & $7.253 \times 10^{-3}$ & $3.395 \times 10^{-2}$ & $1.192 \times 10^{-3}$ & $5.585 \times 10^{-3}$ \\
$\frac{1}{64}$ & $\frac{1}{8}$ & $6.236 \times 10^{-5}$ & $1.124 \times 10^{-3}$ & $8.473 \times 10^{-3}$ & $2.968 \times 10^{-4}$ & $1.374 \times 10^{-3}$ \\
\hline rate $(h)$ & - & 2.00 & 1.34 & 1.00 & 1.00 & 1.00 \\
\hline
\end{tabular}

Table 5

Errors and rates for $\left(W_{h, \mathrm{SD}}\right)\left(\mathrm{FEM} / \mathrm{SIPG} \sigma=18, k_{2}=2\right)$.

Further, we increase the degree of approximation in $\Omega_{2}$ to three. Table 6 shows the errors and convergence rates for this case. We note that the order of approximation remains one in the Stokes domain and increasing the order to three does not have any further improvement in the accuracy of the solution in both domains. This is expected because the order of approximation in $\Omega_{1}$ remains one.

It is clear that the two-grid method performs well for the coupled Stokes and Darcy problem. The real advantage of this method will be shown in the case of the coupled Navier-Stokes and Darcy problem because we avoid solving a non-linear problem on the fine mesh. 


\begin{tabular}{|c|c|c|c|c|c|c|}
\hline$h$ & $H$ & $\left\|\boldsymbol{U}_{h, 1}-\boldsymbol{u}_{1}\right\|_{L^{2}\left(\Omega_{1}\right)}$ & $\left\|P_{h, 1}-p_{1}\right\|_{L^{2}\left(\Omega_{1}\right)}$ & $\left\|\boldsymbol{D}\left(\boldsymbol{U}_{h, 1}-\boldsymbol{u}_{1}\right)\right\|_{L^{2}\left(\Omega_{1}\right)}$ & $\left\|P_{h, 2}-p_{2}\right\|_{L^{2}\left(\Omega_{2}\right)}$ & $\left\|\nabla\left(P_{h, 2}-p_{2}\right)\right\|_{L^{2}\left(\Omega_{2}\right)}$ \\
\hline$\frac{1}{4}$ & $\frac{1}{2}$ & $1.599 \times 10^{-2}$ & $6.417 \times 10^{-2}$ & $1.363 \times 10^{-1}$ & $4.942 \times 10^{-3}$ & $2.328 \times 10^{-2}$ \\
$\frac{1}{16}$ & $\frac{1}{4}$ & $9.926 \times 10^{-4}$ & $7.196 \times 10^{-3}$ & $3.395 \times 10^{-2}$ & $1.191 \times 10^{-3}$ & $5.579 \times 10^{-3}$ \\
$\frac{1}{64}$ & $\frac{1}{8}$ & $6.233 \times 10^{-5}$ & $1.119 \times 10^{-3}$ & $8.470 \times 10^{-3}$ & $2.967 \times 10^{-4}$ & $1.375 \times 10^{-3}$ \\
\hline rate $(h)$ & - & 2.00 & 1.34 & 1.00 & 1.00 & 1.00 \\
\hline
\end{tabular}

Table 6

Errors and rates for $\left(W_{h, \mathrm{SD}}\right)\left(\mathrm{FEM} / \mathrm{NIPG} \sigma=0, k_{2}=3\right)$.

\subsection{Convergence Study for Decoupled Navier-Stokes and Darcy problem}

First we solve the fully coupled problem $\left(W_{\mathrm{NS}}^{h}\right)$ on a sequence of meshes $\mathcal{E}_{h}$. Table 7 shows the errors and convergence rates for the solution from the fully

\begin{tabular}{|c|c|c|c|c|c|}
\hline$h$ & $\left\|\boldsymbol{U}_{1}^{h}-\boldsymbol{u}_{1}\right\|_{L^{2}\left(\Omega_{1}\right)}$ & $\left\|P_{1}^{h}-p_{1}\right\|_{L^{2}\left(\Omega_{1}\right)}$ & $\left\|\boldsymbol{D}\left(\boldsymbol{U}_{1}^{h}-\boldsymbol{u}_{1}\right)\right\|_{L^{2}\left(\Omega_{1}\right)}$ & $\left\|P_{2}^{h}-p_{2}\right\|_{L^{2}\left(\Omega_{2}\right)}$ & $\left\|\nabla\left(P_{2}^{h}-p_{2}\right)\right\|_{L^{2}\left(\Omega_{2}\right)}$ \\
\hline$\frac{1}{4}$ & $1.598 \times 10^{-2}$ & $6.889 \times 10^{-2}$ & $1.366 \times 10^{-1}$ & $2.598 \times 10^{-2}$ & $1.625 \times 10^{-1}$ \\
$\frac{1}{16}$ & $9.988 \times 10^{-4}$ & $6.754 \times 10^{-3}$ & $3.390 \times 10^{-2}$ & $1.592 \times 10^{-3}$ & $3.841 \times 10^{-2}$ \\
$\frac{1}{64}$ & $6.232 \times 10^{-5}$ & $7.403 \times 10^{-4}$ & $8.467 \times 10^{-3}$ & $9.948 \times 10^{-5}$ & $9.541 \times 10^{-3}$ \\
\hline rate $(\mathrm{h})$ & 2.00 & 1.59 & 1.00 & 2.00 & 1.00 \\
\hline
\end{tabular}

Table 7

Errors and rates for $\left(W_{\mathrm{NS}}^{h}\right)\left(\mathrm{FEM} / \mathrm{NIPG} \sigma=1, k_{2}=1\right)$.

coupled Navier-Stokes and Darcy problem solved using a Picard iteration with a tolerance set at $10^{-7}$. We observe order one convergence rate in the energy norm for the Navier-Stokes velocity and Darcy pressure. We then compare the solution from the fully coupled problem to the decoupled Navier-Stokes and Darcy problems $\left(W_{h, \mathrm{NSD}}\right)$. From Table 8 we note that the errors from

\begin{tabular}{|c|c|c|c|c|c|c|}
\hline$h$ & $H$ & $\left\|\boldsymbol{U}_{h, 1}-\boldsymbol{u}_{1}\right\|_{L^{2}\left(\Omega_{1}\right)}$ & $\left\|P_{h, 1}-p_{1}\right\|_{L^{2}\left(\Omega_{1}\right)}$ & $\left\|\boldsymbol{D}\left(\boldsymbol{U}_{h, 1}-\boldsymbol{u}_{1}\right)\right\|_{L^{2}\left(\Omega_{1}\right)}$ & $\left\|P_{h, 2}-p_{2}\right\|_{L^{2}\left(\Omega_{2}\right)}$ & $\left\|\nabla\left(P_{h, 2}-p_{2}\right)\right\|_{L^{2}\left(\Omega_{2}\right)}$ \\
\hline$\frac{1}{4}$ & $\frac{1}{2}$ & $1.594 \times 10^{-2}$ & $9.7277 \times 10^{-2}$ & $1.365 \times 10^{-1}$ & $4.258 \times 10^{-2}$ & $1.708 \times 10^{-1}$ \\
$\frac{1}{16}$ & $\frac{1}{4}$ & $9.696 \times 10^{-4}$ & $1.728 \times 10^{-2}$ & $3.399 \times 10^{-2}$ & $7.068 \times 10^{-3}$ & $4.029 \times 10^{-2}$ \\
$\frac{1}{64}$ & $\frac{1}{8}$ & $7.137 \times 10^{-5}$ & $3.961 \times 10^{-3}$ & $8.477 \times 10^{-3}$ & $1.567 \times 10^{-3}$ & $9.896 \times 10^{-3}$ \\
\hline rate $(h)$ & - & 2.00 & 1.00 & 1.00 & 1.20 & 1.20 \\
\hline
\end{tabular}

Table 8

Errors and rates for $\left(W_{h, \mathrm{NSD}}\right)\left(\mathrm{FEM} / \mathrm{NIPG} \sigma=1, k_{2}=1\right)$.

the two-grid method are comparable to the fully coupled problem for the Navier-Stokes and Darcy velocities in Table 7 . The convergence rates are order one for the velocity and pressure in both domains. This is better than the predicted order $h^{3 / 4}$ for the case $H=\sqrt{h}$. We note that the same improved rate has been reported in $[11,12]$. Next, we increase the order of approximation in $\Omega_{2}$ to $k_{2}=2$. From Table 9 as expected the Darcy solution is more accurate compared to the order one approximation, the convergence rate in the velocity increases to two. In Table 10 we observe that the two grid solution with $k_{2}=2$ is more accurate compared to the one with $k_{2}=1$ in Table 8 . The Darcy velocity error decreases by a factor of almost 4 , this in-turn results in an improvement in the Navier-Stokes velocity and pressure solutions in $\Omega_{1}$. However, due to the fact that the order of approximation in $\Omega_{1}$ is one only, 


\begin{tabular}{|c|c|c|c|c|c|}
\hline$h$ & $\left\|\boldsymbol{U}_{1}^{h}-\boldsymbol{u}_{1}\right\|_{L^{2}\left(\Omega_{1}\right)}$ & $\left\|P_{1}^{h}-p_{1}\right\|_{L^{2}\left(\Omega_{1}\right)}$ & $\left\|\boldsymbol{D}\left(\boldsymbol{U}_{1}^{h}-\boldsymbol{u}_{1}\right)\right\|_{L^{2}\left(\Omega_{1}\right)}$ & $\left\|P_{2}^{h}-p_{2}\right\|_{L^{2}\left(\Omega_{2}\right)}$ & $\left\|\nabla\left(P_{2}^{h}-p_{2}\right)\right\|_{L^{2}\left(\Omega_{2}\right)}$ \\
\hline$\frac{1}{4}$ & $1.599 \times 10^{-2}$ & $6.687 \times 10^{-2}$ & $1.366 \times 10^{-1}$ & $7.449 \times 10^{-3}$ & $1.463 \times 10^{-2}$ \\
$\frac{1}{16}$ & $9.994 \times 10^{-4}$ & $6.685 \times 10^{-3}$ & $3.390 \times 10^{-2}$ & $4.953 \times 10^{-4}$ & $9.275 \times 10^{-4}$ \\
$\frac{1}{64}$ & $6.232 \times 10^{-5}$ & $7.379 \times 10^{-4}$ & $8.467 \times 10^{-3}$ & $3.1225 \times 10^{-5}$ & $5.812 \times 10^{-5}$ \\
\hline rate $(h)$ & 2.00 & 1.59 & 1.00 & 2.00 & 2.00 \\
\hline
\end{tabular}

Table 9

Errors and rates for $\left(W_{\mathrm{NS}}^{h}\right)\left(\mathrm{FEM} / \mathrm{NIPG} \sigma=0, k_{2}=2\right)$.

\begin{tabular}{|c|c|c|c|c|c|c|}
\hline$h$ & $H$ & $\left\|\boldsymbol{U}_{h, 1}-\boldsymbol{u}_{1}\right\|_{L^{2}\left(\Omega_{1}\right)}$ & $\left\|P_{h, 1}-p_{1}\right\|_{L^{2}\left(\Omega_{1}\right)}$ & $\left\|\boldsymbol{D}\left(\boldsymbol{U}_{h, 1}-\boldsymbol{u}_{1}\right)\right\|_{L^{2}\left(\Omega_{1}\right)}$ & $\left\|P_{h, 2}-p_{2}\right\|_{L^{2}\left(\Omega_{2}\right)}$ & $\left\|\nabla\left(P_{h, 2}-p_{2}\right)\right\|_{L^{2}\left(\Omega_{2}\right)}$ \\
\hline$\frac{1}{4}$ & $\frac{1}{2}$ & $1.594 \times 10^{-2}$ & $7.758 \times 10^{-2}$ & $1.364 \times 10^{-1}$ & $2.562 \times 10^{-2}$ & $1.708 \times 10^{-1}$ \\
$\frac{1}{16}$ & $\frac{1}{4}$ & $9.674 \times 10^{-4}$ & $1.278 \times 10^{-2}$ & $3.397 \times 10^{-2}$ & $6.131 \times 10^{-3}$ & $1.057 \times 10^{-2}$ \\
$\frac{1}{64}$ & $\frac{1}{8}$ & $6.698 \times 10^{-5}$ & $2.882 \times 10^{-3}$ & $8.474 \times 10^{-3}$ & $1.511 \times 10^{-5}$ & $2.616 \times 10^{-3}$ \\
\hline rate $(h)$ & - & 2.00 & 1.00 & 1.00 & 1.20 & 1.20 \\
\hline
\end{tabular}

Table 10

Errors and rates for $\left(W_{h, \mathrm{NSD}}\right)\left(\mathrm{FEM} / \mathrm{NIPG} \sigma=0, k_{2}=2\right)$.

the errors in Table 10 are larger compared to the coupled problem in Table 9. The convergence rate for the Navier-Stokes velocity and pressure in $\Omega_{1}$ is order one, the same holds true for the Darcy velocity in $\Omega_{2}$. We also present results for the SIPG method below. As we noted in the case with the Stokes and Darcy coupling the penalty parameter $\sigma$ has to be chosen to be large enough in order to observe the expected convergence rate. Table 11 shows

\begin{tabular}{|c|c|c|c|c|c|}
\hline$h$ & $\left\|\boldsymbol{U}_{1}^{h}-\boldsymbol{u}_{1}\right\|_{L^{2}\left(\Omega_{1}\right)}$ & $\left\|P_{1}^{h}-p_{1}\right\|_{L^{2}\left(\Omega_{1}\right)}$ & $\left\|\boldsymbol{D}\left(\boldsymbol{U}_{1}^{h}-\boldsymbol{u}_{1}\right)\right\|_{L^{2}\left(\Omega_{1}\right)}$ & $\left\|P_{2}^{h}-p_{2}\right\|_{L^{2}\left(\Omega_{2}\right)}$ & $\left\|\nabla\left(P_{2}^{h}-p_{2}\right)\right\|_{L^{2}\left(\Omega_{2}\right)}$ \\
\hline$\frac{1}{4}$ & $1.600 \times 10^{-2}$ & $6.681 \times 10^{-2}$ & $1.366 \times 10^{-1}$ & $5.060 \times 10^{-2}$ & $1.612 \times 10^{-1}$ \\
$\frac{1}{16}$ & $1.000 \times 10^{-3}$ & $6.682 \times 10^{-3}$ & $3.390 \times 10^{-2}$ & $4.000 \times 10^{-4}$ & $3.9791 \times 10^{-2}$ \\
$\frac{1}{64}$ & $6.232 \times 10^{-5}$ & $7.378 \times 10^{-4}$ & $8.467 \times 10^{-3}$ & $2.618 \times 10^{-5}$ & $9.974 \times 10^{-3}$ \\
\hline rate $(h)$ & 2.00 & 1.59 & 1.00 & 2.00 & 1.00 \\
\hline
\end{tabular}

Table 11

Errors and rates for $\left(W_{\mathrm{NS}}^{h}\right)\left(\mathrm{FEM} / \mathrm{SIPG} \sigma=6, k_{2}=1\right)$.

\begin{tabular}{|c|c|c|c|c|c|c|}
\hline$h$ & $H$ & $\left\|\boldsymbol{U}_{h, 1}-\boldsymbol{u}_{1}\right\|_{L^{2}\left(\Omega_{1}\right)}$ & $\left\|P_{h, 1}-p_{1}\right\|_{L^{2}\left(\Omega_{1}\right)}$ & $\left\|\boldsymbol{D}\left(\boldsymbol{U}_{h, 1}-\boldsymbol{u}_{1}\right)\right\|_{L^{2}\left(\Omega_{1}\right)}$ & $\left\|P_{h, 2}-p_{2}\right\|_{L^{2}\left(\Omega_{2}\right)}$ & $\left\|\nabla\left(P_{h, 2}-p_{2}\right)\right\|_{L^{2}\left(\Omega_{2}\right)}$ \\
\hline$\frac{1}{4}$ & $\frac{1}{2}$ & $1.594 \times 10^{-2}$ & $7.931 \times 10^{-2}$ & $1.364 \times 10^{-1}$ & $5.289 \times 10^{-2}$ & $1.661 \times 10^{-1}$ \\
$\frac{1}{16}$ & $\frac{1}{4}$ & $9.747 \times 10^{-4}$ & $1.278 \times 10^{-2}$ & $3.397 \times 10^{-2}$ & $5.714 \times 10^{-3}$ & $4.153 \times 10^{-2}$ \\
$\frac{1}{64}$ & $\frac{1}{8}$ & $6.350 \times 10^{-5}$ & $2.842 \times 10^{-3}$ & $8.474 \times 10^{-3}$ & $1.482 \times 10^{-3}$ & $1.029 \times 10^{-2}$ \\
\hline rate $(h)$ & - & 2.00 & 1.00 & 1.00 & 1.20 & 1.20 \\
\hline
\end{tabular}

Table 12

Errors and rates for $\left(W_{h, \mathrm{NSD}}\right)\left(\mathrm{FEM} / \mathrm{SIPG} \sigma=6, k_{2}=1\right)$.

the errors and convergence rate for the fully coupled problem with $k_{2}=1$ using the SIPG method in $\Omega_{2}$. Table 12 shows the errors and convergence rate from the two grid method on the same meshes. We observe the same trend as in the case with the NIPG method above. The errors for the two-grid method algorithm converge with order one and are comparable to the fully coupled problem.

Tables 13 and 14 show the errors and convergence rates for the fully coupled Navier-Stokes and Darcy model and the two-grid method respectively 


\begin{tabular}{|c|c|c|c|c|c|}
\hline$h$ & $\left\|\boldsymbol{U}_{1}^{h}-\boldsymbol{u}_{1}\right\|_{L^{2}\left(\Omega_{1}\right)}$ & $\left\|P_{1}^{h}-p_{1}\right\|_{L^{2}\left(\Omega_{1}\right)}$ & $\left\|\boldsymbol{D}\left(\boldsymbol{U}_{1}^{h}-\boldsymbol{u}_{1}\right)\right\|_{L^{2}\left(\Omega_{1}\right)}$ & $\left\|P_{2}^{h}-p_{2}\right\|_{L^{2}\left(\Omega_{2}\right)}$ & $\left\|\nabla\left(P_{2}^{h}-p_{2}\right)\right\|_{L^{2}\left(\Omega_{2}\right)}$ \\
\hline$\frac{1}{4}$ & $1.599 \times 10^{-2}$ & $6.604 \times 10^{-2}$ & $1.366 \times 10^{-1}$ & $6.021 \times 10^{-3}$ & $1.301 \times 10^{-2}$ \\
$\frac{1}{16}$ & $9.993 \times 10^{-4}$ & $6.668 \times 10^{-3}$ & $3.390 \times 10^{-2}$ & $3.759 \times 10^{-4}$ & $8.183 \times 10^{-4}$ \\
$\frac{1}{64}$ & $6.233 \times 10^{-5}$ & $7.373 \times 10^{-4}$ & $8.469 \times 10^{-3}$ & $2.349 \times 10^{-5}$ & $5.123 \times 10^{-5}$ \\
\hline rate $(h)$ & 2.00 & 1.59 & 1.00 & 2.00 & 1.00 \\
\hline
\end{tabular}

Table 13

Errors and rates for $\left(W_{\mathrm{NS}}^{h}\right)\left(\mathrm{FEM} / \mathrm{SIPG} \sigma=18, k_{2}=2\right)$.

\begin{tabular}{|c|c|c|c|c|c|c|}
\hline$h$ & $H$ & $\left\|\boldsymbol{U}_{h, 1}-\boldsymbol{u}_{1}\right\|_{L^{2}\left(\Omega_{1}\right)}$ & $\left\|P_{h, 1}-p_{1}\right\|_{L^{2}\left(\Omega_{1}\right)}$ & $\left\|\boldsymbol{D}\left(\boldsymbol{U}_{h, 1}-\boldsymbol{u}_{1}\right)\right\|_{L^{2}\left(\Omega_{1}\right)}$ & $\left\|P_{h, 2}-p_{2}\right\|_{L^{2}\left(\Omega_{2}\right)}$ & $\left\|\nabla\left(P_{h, 2}-p_{2}\right)\right\|_{L^{2}\left(\Omega_{2}\right)}$ \\
\hline$\frac{1}{4}$ & $\frac{1}{2}$ & $1.594 \times 10^{-2}$ & $7.722 \times 10^{-2}$ & $1.364 \times 10^{-1}$ & $2.406 \times 10^{-2}$ & $4.206 \times 10^{-2}$ \\
$\frac{1}{16}$ & $\frac{1}{4}$ & $9.671 \times 10^{-4}$ & $1.249 \times 10^{-2}$ & $3.397 \times 10^{-2}$ & $6.014 \times 10^{-3}$ & $1.042 \times 10^{-2}$ \\
$\frac{1}{64}$ & $\frac{1}{8}$ & $6.764 \times 10^{-5}$ & $2.784 \times 10^{-3}$ & $8.477 \times 10^{-3}$ & $1.503 \times 10^{-3}$ & $2.606 \times 10^{-3}$ \\
\hline rate $(h)$ & - & 2.00 & 1.00 & 1.00 & 1.20 & 1.20 \\
\hline
\end{tabular}

Table 14

Errors and rates for $\left(W_{h, \mathrm{NSD}}\right)\left(\mathrm{FEM} / \mathrm{SIPG} \sigma=18, k_{2}=2\right)$.

if quadratic approximation is used in the subsurface. Increasing the order of approximation in $\Omega_{2}$ to $k_{2}=2$ for the problem $\left(W_{h, \mathrm{NSD}}\right)$ improves the accuracy of the Darcy velocity compared to the $k_{2}=1$. However, we note that the solution to the fully coupled problem with $k_{2}=2$ in the Darcy region is more accurate compared to the two-grid solution for all variables of the model.

One of the applications of this work has been to couple the Navier-Stokes and Darcy solution with a transport equation [21]. Figures 2 and 3 are plots of the

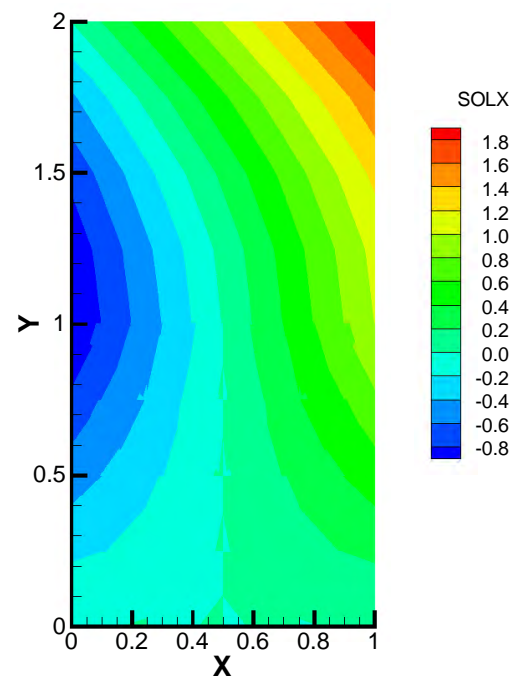

(a) Two Grid

Fig. 2. x- component of velocity $H=0.5, h=0.25, F E M / N I P G, k_{2}=2, \sigma=0$.

$x$ and $y$ components of the velocity solutions respectively for the fully coupled Navier-Stokes/Darcy scheme and the two-grid schemes. It is clear that even for the coarse mesh $H=0.5$ the two-grid solution from $h=0.25$ gives the 


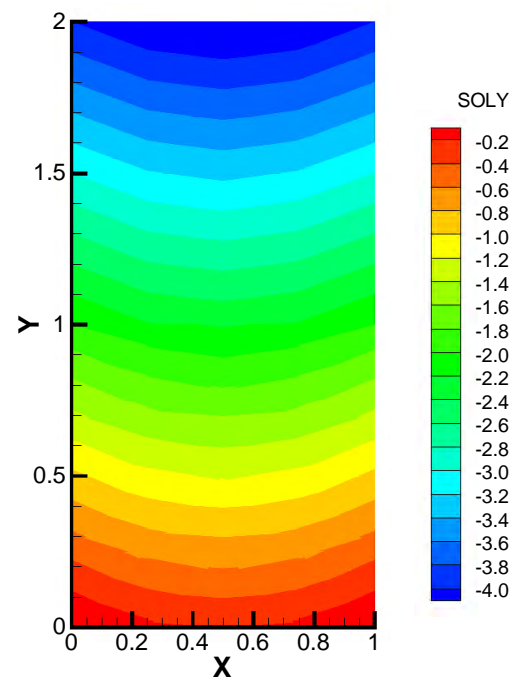

(a) Two Grid

Fig. 3. y- component of velocity $H=0.5, h=0.25, F E M / N I P G, k_{2}=2, \sigma=0$.

same information for the velocity as in the case for the fully coupled problem. We note for the application of this work in tracking contaminants the velocity field for the coupled problem is the input we seek to couple with the transport equation [21].

\subsection{CPU Times: Two-grid Coupled Navier-Stokes and Darcy Model}

The advantage of the two-grid method for the Navier-Stokes and Darcy coupling is that it results in a linearized system. This means that on the fine grid we only solve the linear system resulting from an Oseen problem in $\Omega_{1}$ once. This results in significant gains in computational time and efficiency of the solver. We first present the CPU times for solving the linear systems.

Table 15

\begin{tabular}{|c|c|c|c|c|}
\hline$h$ & $H$ & Fully Coupled $\left(t_{W_{\mathrm{NS}}^{h}}\right)$ & Navier-Stokes $\left(t_{W_{h, \mathrm{NS}}}\right)$ & Darcy $\left(t_{W_{h, D}}\right)$ \\
\hline$\frac{1}{16}$ & $\frac{1}{4}$ & $1.29 \times 10^{0}$ & $6.00 \times 10^{-2}$ & $1.20 \times 10^{-1}$ \\
\hline$\frac{1}{64}$ & $\frac{1}{8}$ & $8.731 \times 10^{+1}$ & $1.69 \times 10^{0}$ & $7.36 \times 10^{0}$ \\
\hline
\end{tabular}

Direct Solver CPU times (s): $W_{\mathrm{NS}}^{h}, W_{h, N S}, W_{h, D},\left(F E M / N I P G \quad \sigma=0, k_{2}=2\right)$.

Table 15 shows the CPU times for the fully coupled Navier-Stokes/Darcy model and the two-grid method. It is very clear that decoupling the problem results in significant reduction in computational time. In most finite element computations the bulk of the time is spent during the assembly of the matrices for the linear system. We also present the total time taken to assemble and solve the system. 


\begin{tabular}{|c|c|c|c|c|c|}
\hline$h$ & $H$ & Fully Coupled $\left(t_{W_{\mathrm{NS}}^{h}}\right)$ & Fully Coupled $\left(t_{W_{\mathrm{NS}}^{H}}\right)$ & Navier-Stokes $\left(t_{W_{h, \mathrm{NS}}}\right)$ & Darcy $\left(t_{W_{h, D}}\right)$ \\
\hline$\frac{1}{16}$ & $\frac{1}{4}$ & $9.4 \times 10^{0}$ & $3.2 \times 10^{-1}$ & $6.0 \times 10^{-1}$ & $1.6 \times 10^{-1}$ \\
\hline$\frac{1}{64}$ & $\frac{1}{8}$ & $1.0 \times 10^{+3}$ & $1.1 \times 10^{0}$ & $2.4 \times 10^{2}$ & $1.1 \times 10^{1}$ \\
\hline
\end{tabular}

Table 16

Assembly and Solve CPU times (s): $W_{\mathrm{NS}}^{h}, W_{h, N S}, W_{h, D},(F E M / N I P G \sigma=0$, $\left.k_{2}=2\right)$.

From Table 16 it is clear that the decoupling technique cuts down the computational time significantly. In a sequential implementation, the two-grid method is almost 9 times faster than the fully coupled approach for a mesh of size $h=1 / 16$. If the mesh is refined and $h=1 / 64$, the two-grid method is about 4 times faster than the fully coupled approach. In that case, we note that the linear system for the fully coupled problem is of size $(77825 \times 77825)$ whereas the decoupled problems are of size $(28673 \times 28673)$ and $(49152 \times 49152)$ for the Oseen and Darcy problems respectively.

\subsection{Case of Impermeable Inclusion in Subsurface}

Next we consider a case for which the exact solution for the coupled flow problem in unknown. The computational domain $\Omega$ is divided into $\Omega_{1}=$ $(0.0,1.5) \times(1.0,2.0)$ and $\Omega_{2}=(0.0,1.5) \times(0.0,1.5)$. The porous medium $\Omega_{2}$ has an enclosure of low permeability $(0.5,1.0) \times(0.25,0.75)$ with $\boldsymbol{K}=10^{-5}$ surrounded by an area with $\boldsymbol{K}=1.0$. We consider the following boundary conditions:

$$
\boldsymbol{u}_{1}(x, y)= \begin{cases}(-(y-2.0)(y-1.0), 0.0), & \text { for } x=0.0 \\ (-0.5(y-2.0)(y-1.0), 0.0), & \text { for } x=1.5 \\ (0.0,0.0) & \text { otherwise }\end{cases}
$$

The Dirichlet and Neumann boundary conditions are chosen to be zero and the data functions $\boldsymbol{f}_{1}$ and $f_{2}$ are also zero. The expected solution is a parabolic flow from left to right in the free flow domain with some fluid percolating into the porous medium. We compare the computational time taken to solve the fully coupled problem and the two-grid method. First we solve the problem using the two-grid method, this requires solving the problem on a coarse mesh. The choice of a coarse mesh for this problem is $H=\frac{1}{8}$. The coarse mesh consists of 854 triangular elements. The choice of MINI elements in $\Omega_{1}$ and the NIPG method of order two with zero penalty in $\Omega_{2}$ results in a fully coupled problem of size $3995 \times 3995$ which takes 6.25 seconds to solve. Next we refine the coarse mesh twice to obtain a fine mesh with 13664 triangular elements $\left(h=\frac{1}{32}\right)$. On the fine mesh we solve a decoupled Navier-Stokes problem of size $26935 \times 26935$ and a Darcy problem of size $36000 \times 36000$. It 
takes $2.12 \times 10^{2}$ seconds to solve the resulting decoupled problems. In Figure

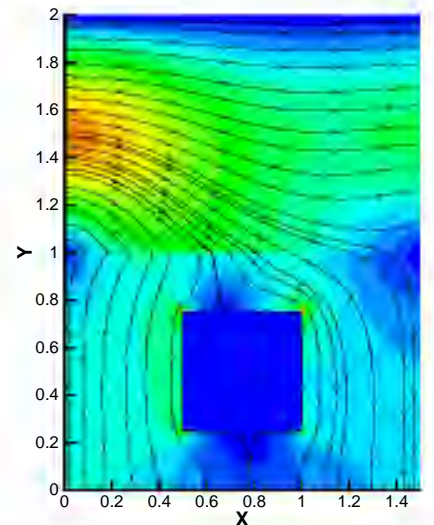

(a) Two Grid

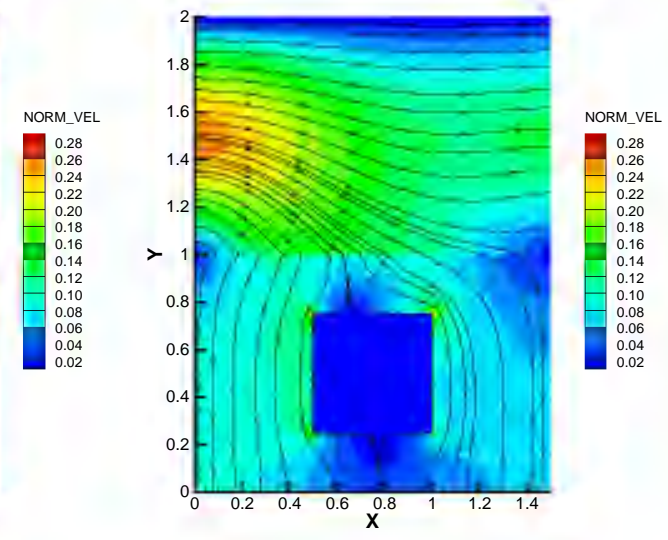

(b) Fully Coupled

Fig. 4. Euclidian norm of velocity $H=\frac{1}{8}, h=\frac{1}{32}, F E M / N I P G, k_{2}=2, \sigma=0$.

4 we compare the solutions obtained from solving the fully coupled problem on the fine mesh. It is clear that both solutions capture the flow well and are comparable. The fully coupled problem for this case takes $1.904 \times 10^{3}$ seconds to solve. The two-grid method is 8 times faster than the fully coupled method in this example and gives an accurate solution.

\section{Conclusion}

We have presented a two-grid method for the coupled Navier-Stokes and Darcy problem. The method naturally yields decoupled and linear problems for the surface and subsurface regions. We have shown that the two-grid method is computationally efficient: the accuracy of the solution is comparable to fully coupled solution, and the CPU times are significantly smaller. Using the flexibility of discontinuous Galerkin methods in the subsurface, we obtained a more accurate solution by locally increasing the order of approximation.

\section{References}

[1] V. Girault, B. Rivière, DG approximation of coupled Navier-Stokes and Darcy equations by Beavers-Joseph Saffman interface condition, SIAM Journal on Numerical Analysis 47 (2009) 2052-2089.

[2] M. Discacciati, E. Miglio, A. Quarteroni, Mathematical and numerical models for coupling surface and groundwater flows, Appl. Numer. Math. 43 (2001) 57-74. 
[3] L. Badea, M. Discacciati, A. Quarteroni, Mathematical analysis of the Navier-Stokes and Darcy coupling, Technical Report of Politecnico di Milano.

[4] P. Chidyagwai, B. Rivière, On the solution of the coupled Navier-Stokes and Darcy equations, Computer Methods in Applied Mechanics and Engineering. 198 (2009) 3806-3820.

[5] B. Rivière, Analysis of a discontinuous finite element method for the coupled Stokes and Darcy problems, Journal of Scientific Computing 22 (2005) 479-500.

[6] B. Rivière, I. Yotov, Locally conservative coupling of Stokes and Darcy flow, SIAM J. Numer. Anal. 42 (2005) 1959-1977.

[7] E. Burman, P. Hansbo, A unified stabilized method for Stokes and Darcy's equations, J. Computational and Applied Mathematics 198 (1) (2007) 35-51.

[8] M. Discacciati, A. Quarteroni, A. Valli, Robin-Robin domain decomposition methods for the Stokes-Darcy coupling, SIAM J. Numer. Anal. 45 (3) (2007) 1246-1268.

[9] M. Discacciati, A. Quarteroni, Analysis of a domain decomposition method for the coupling of Stokes and Darcy equations, in: B. et al (Ed.), Numerical Analysis and Advanced Applications - ENUMATH 2001, Springer, Milan, 2003, pp. 3-20.

[10] N. Hanspal, A. Waghode, V. Nassehi, R. Wakeman, Numerical analysis of coupled Stokes/Darcy flows in industrial filtrations, Transport in Porous Media 64 (1) (2006) 73-101.

[11] M. Mu, J. Xu, A Two grid method of a mixed Stokes-Darcy model for coupling fluid flow with porous media flow, SIAM J. Numerical Analysis 45 (2007) 1801-1813.

[12] M. Cai, M. Mu, J. Xu, Numerical solution to a mixed NavierStokes/Darcy model by the two-grid approach, SIAM Journal on Numerical Analysis 47 (5) (2009) 3325-3338.

[13] G. Beavers, D. Joseph, Boundary conditions at a naturally permeable wall, Journal of Fluid Mechanics 30 (1967) 197-207.

[14] W. Jager, A. Mikelic, On the boundary conditions at the contact interface between a porous medium and a free fluid, Ann. Scuola Norm. Sup. Pisa Cl.Sci. 23 (1996) 403-465.

[15] P. Saffman, On the boundary condition at the surface of a porous medium, Journal of Fluid Mechanics 2 (1971) 93-101.

[16] P. Ciarlet, The finite element method for elliptic problems, NorthHolland, Amsterdam, 1978.

[17] D. Arnold, F. Brezzi, M. Fortin, A stable finite element for the Stokes equations, Estratto da Calcolo XXI.

[18] P. Chidyagwai, B. Rivière, Numerical modelling of coupled surface and subsurface flow systems, Advances in Water Resources 33 (1) (2010) 92 $-105$.

[19] B. Rivière, Discontinous Galerkin Method for Solving Elliptic and 
Parabolic Equations: Theory and Implementation, Society for Industrial and Applied Mathematics, 2008.

[20] Y. Epshteyn, B. Rivière, Estimation of penalty parameters for symmetric interior penalty Galerkin methods, Journal of Computational and Applied Mathematics 206 (2007) 843-872.

[21] A. Cesmelioglu, P.Chidyagwai, B. Rivière, Continuous and Discontinuous Finite Element Methods for Coupled Surface-Subsurface Flow and Transport Problems, Applied Numerical Mathematics.Submitted. 